\title{
Dinámica poblacional y relaciones con el hábitat de Aphanius iberus (Valenciennes 1846) en un humedal con salinas en explotación
}

Raquel Moreno-Valcárcel ${ }^{1,2}$, Mar Torralva², David Verdiell-Cubedo², Ana Ruiz-Navarro² \& Francisco José OlivaPaterna ${ }^{2}$

1 Grupo Aphanius. Departamento de Zoología. Universidad de Córdoba, 14071, Córdoba, España.

2 Departamento de Zoología y Antropología Física. Universidad de Murcia, 30100, Murcia, España.

Correspondencia

F.J. Oliva-Paterna

E-mail: fjoliva@um.es

Recibido: 6 noviembre 2012

Aceptado: 7 diciembre 2012

Publicado on-line: 14 diciembre 2012

\section{Resumen}

Aphanius iberus es un ciprinodóntido endémico de la Península Ibérica muy amenazado, a pesar de los esfuerzos de gestión realizados. Se presenta la variación temporal de su abundancia y su relación con el hábitat, en un humedal con salinas en explotación. La densidad muestra una estacionalidad con máximos durante el verano y otoño. Cambios en los niveles de agua de la balsa provocan incrementos en la salinidad que pueden haber ejercido efectos negativos sobre su abundancia relacionados con la reducción de la vegetación acuática. Se observaron cambios en el uso del microhábitat que pueden relacionarse con el ciclo temporal de abundancia y del mesohábitat. La abundancia mostró una relación con la vegetación acuática y la cercanía a motas salineras, áreas probablemente usadas como refugio. Los resultados apoyan la necesidad de criterios biológicos en la gestión de humedales para mantener la especie. Los efectos observados deben considerarse en acciones de conservación sobre sus poblaciones.

Palabras clave: Cyprinodontidae, Dinámica poblacional, Microhábitat, Especies amenazadas.

\begin{abstract}
Population dynamic and habitat relationships of Aphanius iberus (Valenciennes 1846) in a wetland managed for salt exploitation

Aphanius iberus is an endemic cyprinodontid of the Iberian Peninsula highly endangered despite management efforts. We present its population dynamic and relationships with the habitat in a wetland managed for salt exploitation. Density showed a seasonal pattern characterized by maxima values during summer and autumn. Changes in water level that drastically increased the salinity should have produced effects on fish abundance by reducing vegetation mats. The species showed changes in microhabitat use which could be linked with its seasonal abundance and the environmental conditions, at mesohabitat scale. Its abundance showed relationships with vegetation mats and the proximity to embankments, areas probably used as a refuge. Results support the need of the biological criteria in the management of wetland in order to maintain populations of this species. The observed effects should be considered when such populations are exposed to recovery actions.
\end{abstract}

Key words: Cyprinodontidae, Population dynamic, Microhabitat, Endangered species. 


\section{Introducción}

Aphanius iberus (Valenciennes 1846) es un ciprinodóntido endémico de la Península Ibérica cuya distribución se restringe a unas pocas poblaciones del litoral Mediterráneo de la Península Ibérica (Doadrio 2002). Esta especie está catalogada como en peligro de extinción (UICN 2012) siendo la pérdida de hábitats y la contaminación química $o$ biológica sus principales factores de amenaza. Aunque se trata de una especie ubiquista que puede ocupar una alta variedad de hábitats, en la actualidad su distribución está mayormente restringida a ambientes salinos o hipersalinos (Fernández-Delgado et al. 1999, Oliva-Paterna et al. 2006, Alcaraz et al. 2008). Además, los humedales con explotaciones salineras, junto con algunos sistemas acuáticos artificiales (Casas et al. 2011), se conforman como hábitats actualmente importantes para la viabilidad de la especie en el sureste peninsular (Oliva-Paterna \& Torralva 2008, OlivaPaterna et al. 2009), albergando poblaciones de la especie con un alto interés conservacionista (Oliva-Paterna et al. 2006).

El conocimiento de la biología y ecología de A. iberus se considera una herramienta básica para su gestión y conservación (Elvira 1998). De hecho, en los últimos años han sido publicados múltiples trabajos sobre aspectos relacionados con su estrategia de vida, genética poblacional e interacción con otras especies (Rincón et al. 2002, Caiola \& De Sostoa 2005, Alcaraz \& García-Berthou 2007, Araguas et al. 2007, Oliva-Paterna et al. 2009, entre otros). Sin embargo, los estudios que incorporan aspectos sobre las relaciones de la especie con su hábitat son escasos (Alcaraz et al. 2008, Casas et al. 2011, Verdiell-Cubedo et al. 2012). Estos estudios adquieren gran importancia a la hora de realizar actuaciones de gestión, ya que en muchos casos, la recuperación de esta especie pasa por la rehabilitación y restauración de ambientes en su rango de distribución nativo (Oliva-Paterna 2006).

En el presente trabajo, se estudia el ciclo de vida de una población de $A$. iberus establecida en las Salinas de Marchamalo. Esta población se ha mostrado como una de las más importantes en términos de abundancia relativa dentro de la unidad ecogeográfica del Mar Menor y Humedales de su entorno (Oliva-Paterna \& Torralva 2008). Durante un periodo superior a dos años, se analizan las re- laciones de la abundancia relativa de la especie con variables limnológicas del sistema a dos escalas de hábitat. En este contexto, los objetivos concretos han sido (i) caracterizar la dinámica poblacional de $A$. iberus en términos de abundancia relativa, (ii) determinar la relación de dicha dinámica con las variables del hábitat (a nivel de mesohábitat), y (iii) establecer las relaciones múltiples existentes entre las variables de microhábitat y la abundancia de la especie. La obtención de estos objetivos nos ayuda en el establecimiento de criterios biológicos útiles en la gestión conservacionista de humedales con salinas en explotación.

\section{Material y métodos}

\section{Área de estudio}

Las Salinas de Marchamalo (UTM 30SYG06) se localizan en la ribera sur del Mar Menor (Murcia, SE España) (Fig. 1). Es una pequeña explotación salinera ubicada en la zona de antiguas lagunas temporales que se encontraban limitadas por los arenales de La Manga. Están clasificadas como humedal con salinas costeras según el inventario de humedales de la Región de Murcia (Esteve et al. 1995). Esta tipología se caracteriza por el mantenimiento de un gradiente de salinidad, que se traduce en una alta heterogeneidad espacial. Las primeras balsas de recepción de agua se encuentran inundadas permanentemente y suelen mostrar salinidades promedio entre 40 y $50 \mathrm{~g} / 1$.

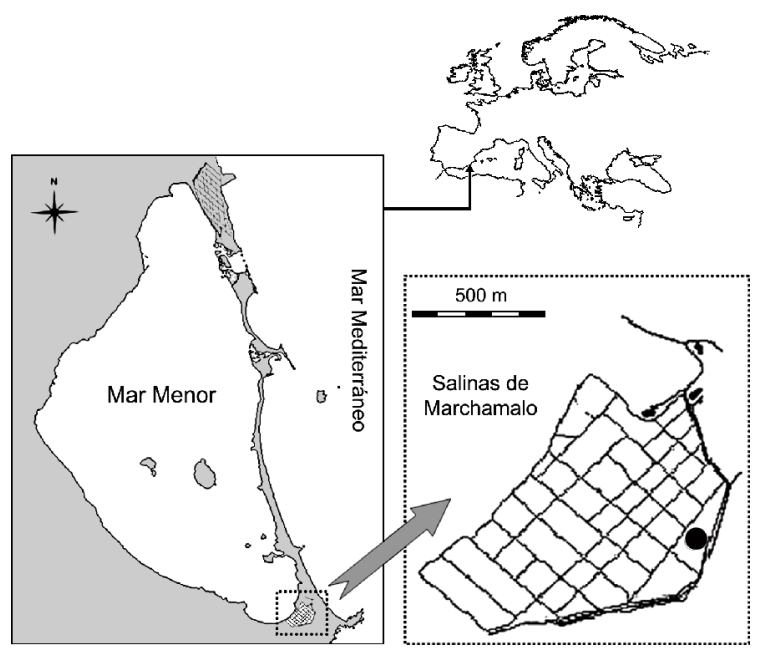

Figura 1. Localización de las Salinas de Marchamalo y situación específica de la localidad de muestreo establecida en las salinas (punto negro).

Figure 1. Location of Marchamalo wetland and sampling pool (black point). 
En el presente estudio, la localidad de muestreo seleccionada fue la primera balsa de recepción (Fig. 1). Se seleccionó esta balsa por mostrar una elevada abundancia de la especie y una elevada proliferación de vegetación acuática, en consecuencia, una mayor heterogeneidad de hábitat disponible para la especie (Oliva-Paterna et al. 2009). Entre la vegetación acuática presente son destacables las manchas de Ruppia spp. y la acumulación temporal de algas filamentosas flotantes como Cladophora spp.

La comunidad de peces presente en la localidad de estudio es prácticamente monoespecífica, siendo A. iberus la especie dominante (OlivaPaterna 2006). No obstante, individuos aislados de las Familias Mugilidae y Blennidae han sido detectados en otros estudios (Ruiz-Navarro et al. 2007, Oliva-Paterna et al. 2009).

\section{Periodo y metodología de captura}

El periodo de estudio abarcó desde marzo de 2006 hasta mayo de 2008. Fueron realizados dos muestreos por cada estación del año, con un total de 20 campañas de muestreo: 8 en primavera, 4 en verano, 4 en otoño y 4 en invierno.

Para la captura de los ejemplares fueron empleadas trampas tipo minnow-trap (Harrison et al. 1986) y trampas de polietileno. Las minnow-traps son trampas de malla metálica de doble embudo forradas con tela de luz de malla de $1 \mathrm{~mm}$. Las trampas de polietileno consisten en trampas fabricadas a partir de botellas de agua, cuya parte superior es cortada y colocada de forma invertida (Fouilland \& Fossati 1996). Estos métodos se han estimado como óptimos para la captura selectiva de peces pequeños que viven en zonas someras (Clavero et al. 2006).

El número de trampas utilizadas fue de 20 a 25 por campaña de muestreo (10-15 minnow-traps y 10 trampas de polietileno). Éstas se distribuían por la totalidad de la localidad de muestreo con un esfuerzo de pesca de 20 a $24 \mathrm{~h}$. Se registró in situ el número total de individuos capturados por trampa y se determinó el sexo de cada individuo; la diferenciación de machos, hembras e indeterminados (individuos juveniles e inmaduros) mediante caracteres morfológicos externos no resulta complicada. Los ejemplares eran devueltos al medio con una mortalidad total a lo largo del estudio inferior al 3\% de las capturas.

\section{Variables de hábitat}

Durante los dos ciclos anuales de estudio se realizó mediante la toma de medidas in situ un seguimiento de variables de hábitat condicionantes o explicativas de la variación en abundancia de la especie en el humedal (Oliva-Paterna et al. 2009). A escala de mesohábitat la unidad de referencia ha sido la localidad o balsa salinera en su conjunto (superficie aproximada de 2 ha). Las variables seleccionadas fueron temperatura del agua $\left({ }^{\circ} \mathrm{C}\right)$, salinidad $(\mathrm{g} / \mathrm{l})$, conductividad $(\mathrm{mS} / \mathrm{cm})$, profundidad $(\mathrm{cm})$ y recubrimiento o cobertura vegetal (\%). En cada muestreo se tomaron cinco registros (réplicas) de temperatura, salinidad y conductividad mediante el uso de un medidor multiparamétrico WTW Multi 350i ${ }^{\circledR}$. Las réplicas fueron tomadas a intervalos de $20 \mathrm{~m}$ a $10 \mathrm{~cm}$ de profundidad y en una franja horaria similar (entre las 12 y $14 \mathrm{~h}$ ). Para la estima de la profundidad $(\mathrm{cm})$ se realizaron entre 20-25 puntos de medida repartidos homogéneamente y abarcando la totalidad de la superficie de la balsa. Finalmente, para determinar el porcentaje de recubrimiento o cobertura en la balsa, en cada punto de toma de profundidad se realizaba una estima visual de la misma en un diámetro de $5 \mathrm{~m}$.

En el análisis realizado con respecto al uso de microhábitat, la unidad de muestreo ha sido el espacio inmediato alrededor de cada trampa (unidad de microhábitat $=$ círculo de $1 \mathrm{~m}$ de diámetro, siendo la trampa el punto medio). Las variables estudiadas a esta escala han sido profundidad, distancia a la orilla $(\mathrm{cm})$, cobertura vegetal, volumen de la vegetación, granulometría y heterogeneidad del sustrato. Se procuró que la distancia entre trampas fuera mayor a $20 \mathrm{~m}$ para evitar la interacción entre unidades de muestreo. La toma de profundidad y distancia perpendicular a la orilla más próxima se realizó en el punto exacto de colocación de la trampa. El porcentaje de recubrimiento o cobertura vegetal fue estimado visualmente para cada unidad de microhábitat. De igual modo, se evaluó el volumen o densidad de la vegetación con las categorías ordinales de 1 (poco denso) a 5 (muy denso) según la proporción de la columna de agua ocupada por dicha vegetación. En lo referente a la granulometría, se realizó una estima visual de las siguientes categorías de sustrato: limo $(\varnothing<0,06 \mathrm{~mm})$, arena $(\varnothing=0,06-1 \mathrm{~mm})$, gravas $(\varnothing=2-15 \mathrm{~mm})$, cantos $(\varnothing=16-63 \mathrm{~mm})$ y guijarros ( $>63 \mathrm{~mm})$. Además, se asignó un valor de 1 a 5 
a cada categoría calculándose la granulometría como el valor promedio y la heterogeneidad de sustrato como la desviación estándar de ésta (Verdiell-Cubedo et al. 2012).

\section{Análisis de datos}

Se ha estudiado la evolución temporal de la abundancia relativa ( $=$ densidad relativa) de la especie mediante las capturas por unidad de esfuerzo (CPUE). Asumiendo que la probabilidad de captura de los individuos es constante, las CPUE son un buen indicador de cambios relativos en el tamaño de una población de peces cuando no es posible conocer la densidad absoluta de la misma. Además, este indicador ha sido utilizado con la especie objetivo y ha mostrado resultados satisfactorios en estudios previos (Oliva-Paterna et al. 2009), así como con otras especies del género Aphanius (Clavero et al. 2007). En el presente trabajo, las CPUEs corresponden con el número de individuos capturados en cada trampa durante 24 horas de pesca $(C P U E=n /$ trampa/24h). Se ha realizado un análisis del patrón temporal de abundancia relativa en machos, hembras e indeterminados en función de los datos procedentes de minnowtraps y trampas de polietileno. Pruebas de comparación de medias han sido aplicadas para la comparación de datos temporales o grupos (machos, hembras) dentro de un mismo periodo.

Para el establecimiento de periodos o fases temporales con cierta estabilidad en las condiciones limnológicas de la balsa (escala mesohábitat), aspecto que puede justificar una aproximación diferencial a las relaciones entre la abundancia relativa y el microhábitat, se han aplicado métodos de reducción de datos (Quinn \& Keough 2002). El método seleccionado ha sido el Análisis de Componentes Principales (ACP) sobre matrices de campañas de muestreo $\mathrm{x}$ variables de mesohábitat con el método Varimax de rotación (Visauta-Vinacua 1997). Las diferencias entre los grupos de campañas de muestreo o fases temporales establecidos mediante el ACP se han comprobado mediante la realización de un Análisis de la Varianza (ANOVA; $\mathrm{p}<0,05$ ) con los valores de puntuación en cada componente de las campañas de muestreo.

El primer paso en el estudio del efecto múltiple del microhábitat sobre la abundancia fue analizar las relaciones bivariantes entre las variables descriptoras del microhábitat y la abundancia rela- tiva de machos, hembras o indeterminados (variables dependientes) mediante Análisis de Regresión (Quinn \& Keough 2002). Las variables fueron transformadas en función de las necesidades del análisis $[\operatorname{Ln}(\mathrm{x}+1)$ o $\operatorname{Arcsen} \sqrt{\mathrm{x}}]$. A continuación, la relación múltiple entre la abundancia y las variables ambientales de microhábitat se estudió mediante Análisis de Regresión Lineal Múltiple por el método de pasos sucesivos (variable dependiente CPUE de machos, hembras e indeterminados). Este procedimiento estadístico selecciona las variables ambientales que tienen más relación con la densidad de la especie. La colinealidad de las variables introducidas en el modelo se ha estudiado mediante el Índice de Tolerancia (Quinn \& Keough 2002). Este análisis del efecto del microhábitat fue realizado en función de las capturas obtenidas con los dos métodos utilizados minnowtraps y trampas de polietileno.

Los análisis estadísticos se han llevado a cabo mediante el paquete estadístico SPSS ${ }^{\circledR}$ v. 15.0 con un nivel de significación estadística de $\mathrm{p}<0,05$.

\section{Resultados}

\section{Ciclo de vida: Abundancia relativa}

La abundancia relativa de la especie ha variado temporalmente de forma significativa a lo largo del año en el caso de adultos (machos y hembras) (Kruskal-Wallis $\mathrm{H}=129,6$; $\mathrm{gl}=19$; $\mathrm{p}<0,001$ ) e indeterminados $(\mathrm{H}=60,6 ; \mathrm{gl}=19 ; \mathrm{p}<0,001)$ (Fig. 2).

Las capturas de individuos adultos han sido significativamente mayores en las minnow-traps que en las trampas de polietileno (valores promedio; minnow-traps $\mathrm{CPUE}=65,81 \pm 15,21$; trampas de polietileno CPUE $=5,65 \pm 1,96)$. En el caso de los indeterminados, la diferencia en la capturabilidad entre tipos de trampa no fue significativa (minnow-traps CPUE $=1,00 \pm 0,50$; trampas de polietileno CPUE $=1,40 \pm 0,63$ ). No obstante, la proporción indeterminados/adultos en las capturas fue superior en trampas de polietileno (minnowtraps 1:38,7; trampas de polietileno 1:1,65). De este modo, a la hora de establecer patrones en la dinámica temporal en los individuos indeterminados de la población se han considerado mayormente los resultados obtenidos mediante trampas de polietileno, sin embargo, en el caso de la dinámica de adultos resultan más significativos los datos obtenidos con minnow-traps. 


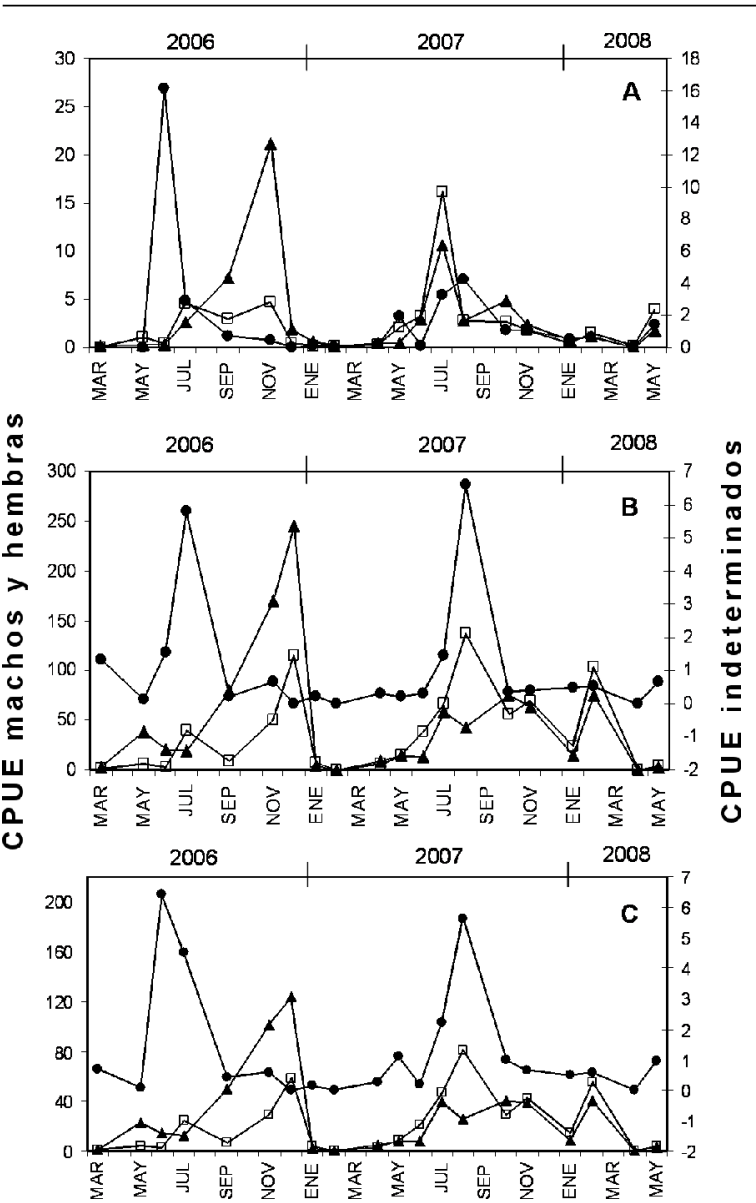

Figura 2. Variación temporal en las capturas por unidad de esfuerzo (CPUEs) de machos (cuadrados blancos), hembras (triángulos negros) e indeterminados (círculos negros) de Aphanius iberus obtenidas con trampas de polietileno (A), con minnow-traps (B) y con ambas metodologías (C).

Figure 2. Temporal changes in catches per unit of effort (CPUEs) of males (white squares), females (black triangles) and indeterminates (black circles) of Aphanius iberus obtained in polyethylene traps (A), minnow-traps (B) and in both methods (C).

A pesar de las diferencias en capturabilidad, el patrón de variación temporal de la abundancia relativa de la especie se mostró similar con los datos obtenidos de ambas metodologías (Fig. 2). La densidad de adultos de A. iberus ha mostrado un patrón temporal con aumentos significativos durante las estaciones de verano y otoño. No obstante, este periodo de incremento poblacional (juniodiciembre) presentó diferencias entre los años 2006 y 2007. Además, los valores promedio de adultos durante este periodo mostraron diferencias significativas entre ambos ciclos (datos minnowtraps; Mann-Whitney $\mathrm{U}=686,0 ; \mathrm{p}<0,001)$.

Aunque la presencia de indeterminados es constante a lo largo del periodo de estudio $(>80 \%$ de las campañas), las densidades relativas más elevadas de éstos se detectan desde los meses de mayo-junio hasta octubre-noviembre. Los valores máximos de indeterminados han sido detectados en junio de 2006 (CPUE=16,17) y agosto de 2007 (CPUE=4,28) (Fig. 2A). No obstante, los valores promedio del ciclo de reclutamiento de indeterminados no han resultado diferentes de forma significativa entre ambos ciclos (datos obtenidos con trampas de polietileno; $\mathrm{U}=769,0 ; \mathrm{p}=0,128)$.

En el caso de las hembras, el ciclo 2006 muestra un incremento en densidad muy patente al final del periodo de incremento poblacional, mostrando máximos de abundancia en noviembre $(\mathrm{CPUE}=170,03)$ y diciembre $(\mathrm{CPUE}=245,78) . \mathrm{Sin}$ embargo, en el ciclo 2007 las hembras no mostraron máximos tan marcados, con un periodo de valores similares entre julio y noviembre (Fig. 3B). Por otro lado, los machos han mostrado un patrón similar a las hembras en el primer ciclo (2006) con valores máximos en diciembre (CPUE= 115,67), si bien, en el segundo ciclo el máximo de abundancia se detectó en uno de los primeros meses de este periodo de incremento poblacional (agosto 2007; CPUE=137,97) (Fig. 2B).

\section{Variables de mesohábitat}

En la figura 3 se refleja el patrón de variación temporal de las variables descriptoras de las condiciones limnológicas de la balsa de estudio (escala de mesohábitat).

La variación de la temperatura del agua ha seguido el patrón anual típico de ambientes mediterráneos, con aumento de las temperaturas durante la primavera, valores altos hasta principios de otoño y mínimos de diciembre a febrero. Los valores máximos se observaron en el mes de junio de $2006\left(32,4^{\circ} \mathrm{C}\right)$ y en mayo de $2007\left(28,4^{\circ} \mathrm{C}\right)$. Los mínimos de temperatura se detectaron en diciembre de $2006\left(14,3{ }^{\circ} \mathrm{C}\right)$ y enero de $2007\left(13,2{ }^{\circ} \mathrm{C}\right)$ (Fig. 3A). Únicamente en tres campañas de muestreo se registraron temperaturas inferiores a $15^{\circ} \mathrm{C}$.

En lo referente a la salinidad y conductividad, durante el periodo de estudio la localidad de muestreo ha presentado unos valores medios de $52,7 \pm 8,2 \mathrm{~g} / 1$ y $82,5 \pm 15,0 \mathrm{mS} / \mathrm{cm}$, respectivamente. Al menos, se han detectado tres picos con máximos en los meses de junio de 2006 (77,1 g/l; $107,0 \mathrm{mS} / \mathrm{cm})$, mayo $(82,7 \mathrm{~g} / 1 ; 113,0 \mathrm{mS} / \mathrm{cm})$ y julio de 2007 (66,4 g/l; 96,7 mS/cm) (Fig. 3B).

En cuanto a la variación en profundidad de la lámina de agua, se han observado disminuciones drásticas que se traducen en mínimos en los meses de junio de $2006(16,5 \mathrm{~cm})$ y enero de 2008 $(14,33 \mathrm{~cm})$ (Fig. 3C). 
Los valores de recubrimiento vegetal para la totalidad de la balsa han variado de forma correlacionada con la profundidad (Correlación de Spearman; Recubrimiento-Profundidad $\mathrm{R}=0,47$; $\mathrm{p}=$ 0,036). A su vez, la profundidad se mostró correlacionada con la temperatura (Profundidad-Temperatura $\mathrm{R}=0,50 ; \mathrm{p}=0,024)$, presentándose los valores más elevados de recubrimiento, profundidad y temperatura durante los meses más cálidos. El valor máximo de cobertura vegetal alcanzado ha sido en junio de 2007 (48,75\%), y el mínimo en abril de 2008 (2,92\%) (Fig. 3D).

En la Tabla 1 se muestran las correlaciones existentes entre las variables de mesohábitat y la abundancia relativa (CPUEs) de machos, hembras e indeterminados. Para individuos adultos (datos de CPUEs obtenidos con minnow-traps) no se han obtenido correlaciones significativas. Únicamente en el caso de los indeterminados (datos de CPUEs obtenidos con trampas de polietileno), se encontró una correlación positiva con los valores de temperatura del agua $(\mathrm{R}=0,53)$ (Tabla 1$)$.

\section{Relaciones con el microhábitat}

Con la finalidad de determinar periodos temporales estables en las condiciones del mesohábitat que pueden afectar la relación del microhábitat con la abundancia de la especie, se realizó un Análisis de Componentes Principales (ACP) sobre la matriz campañas $\mathrm{x}$ variables de mesohábitat. El ACP mostró dos componentes (CPs) con valores propios mayores a 1. El CP-1 ordenó las campañas de muestreo de forma altamente correlacionada con la salinidad $(0,992)$ y conductividad $(0,991)$ de la balsa, mientras que la profundidad $(0,856)$ y temperatura $(0,761)$ son las variables que presentaron mayor correlación con el CP-2 (Tabla 2).

En la Figura 4 se representan las campañas de muestreo ordenadas en función de las puntuaciones obtenidas en el ACP. Éstas reflejan la segregación de un grupo de tres campañas (marzo y junio de 2006, abril de 2007) en la parte derecha de la dispersión de datos (Fig. 4), las cuales presentan salinidades anormalmente elevadas consecuencia de restricciones en la entrada de agua a la balsa salinera y, por tanto, coincidentes con descensos en la profundidad de la lámina de agua (Fig. 3). No obstante, las posibles agrupaciones de campañas de muestreo en función del CP-1 no presentan interpretación temporal cíclica y tampoco mues-
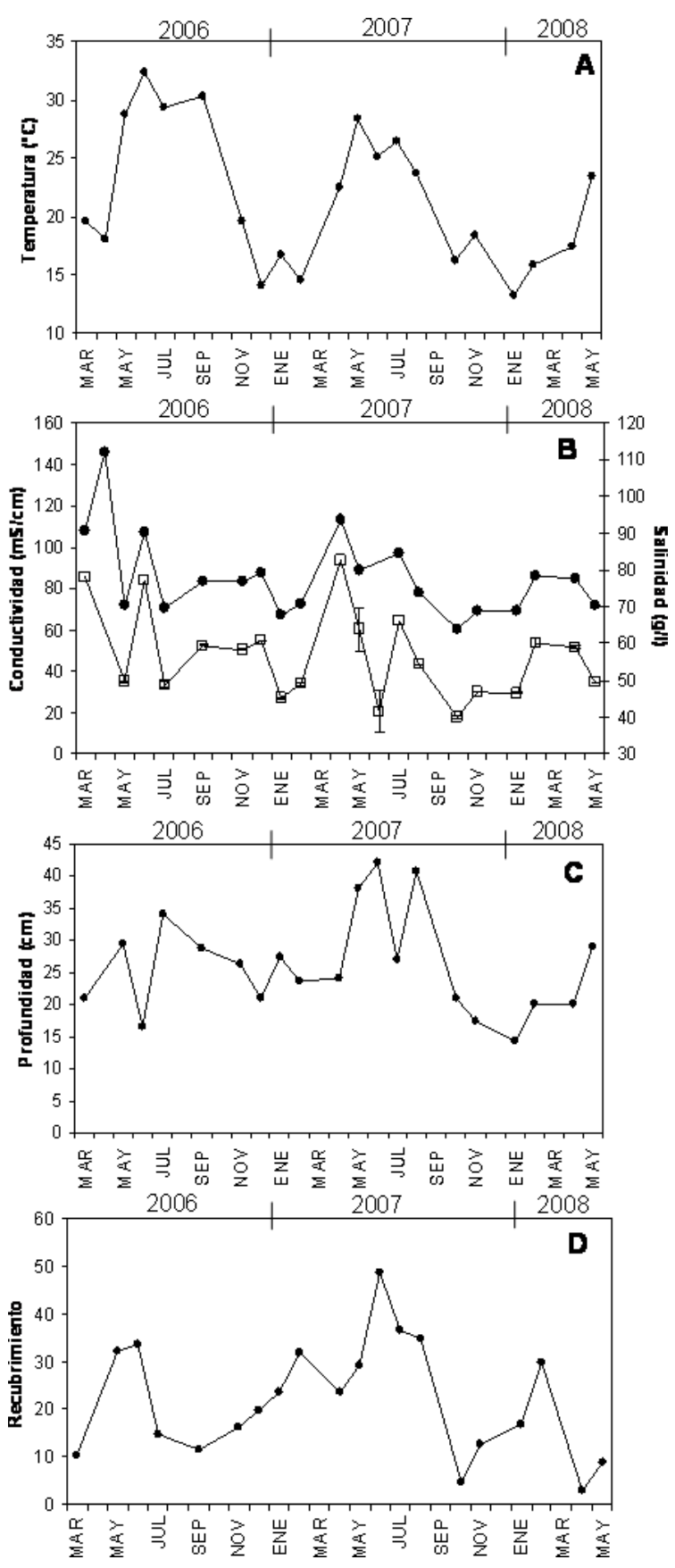

Figura 3. Variación temporal de las variables de mesohábitat en las Salinas de Marchamalo. A. Temperatura B. Conductividad (círculos negros) y salinidad (cuadrados blancos). C. Profundidad. D. Recubrimiento vegetal.

Figure 3. Temporal variation in mesohabitat variables of the Marchamalo wetland. A. Temperature. B. Conductivity (black circles) and salinity (white squares). C. Water depth. D. Vegetation cover.

tran correlación con el ciclo de abundancia de la especie.

Por otro lado, a partir de la ordenación de las campañas de muestreo atendiendo al eje CP2,pueden diferenciarse dos grupos (Periodos 1 y 2 en Fig. 4) de campañas de muestreo segregadas básicamente por diferencias en la temperatura, 
profundidad y recubrimiento de vegetación. Un grupo 1 con campañas localizadas principalmente en la parte positiva del eje CP-2 que, aunque abarca campañas con un gran rango de salinidades, éstas presentan los valores promedio más elevados de profundidad $(30,2 \pm 8,2 \mathrm{~cm})$, temperatura $(26,7 \pm$ $\left.3,8{ }^{\circ} \mathrm{C}\right)$ y recubrimiento $(28,3 \pm 4,14 \%)$. El grupo 2 , con valores situados en la parte negativa del eje CP-2, estaría conformado por campañas con menor profundidad de la lámina de agua $(22,0 \pm 4,4$ $\mathrm{cm})$, temperatura más baja $\left(17,0 \pm 2,9^{\circ} \mathrm{C}\right)$ y menor recubrimiento $(16,1 \pm 3,2 \%)$. Además, estos dos grupos de campañas de muestreo o fases temporales se diferencian significativamente en función de las puntuaciones obtenidas para CP-2 (ANOVA $\mathrm{F}=24,017 ; \mathrm{p}<0,001)$.

Adicionalmente, cuando comparamos estas dos fases temporales con la abundancia relativa de la especie, podemos detectar un alto nivel de coincidencia con su ciclo temporal. El grupo o fase temporal 1 coincide con el periodo de capturas más elevadas de indeterminados o reclutas, lo que puede identificarse como época de reclutamiento (abril a septiembre de 2006 y mayo a agosto de

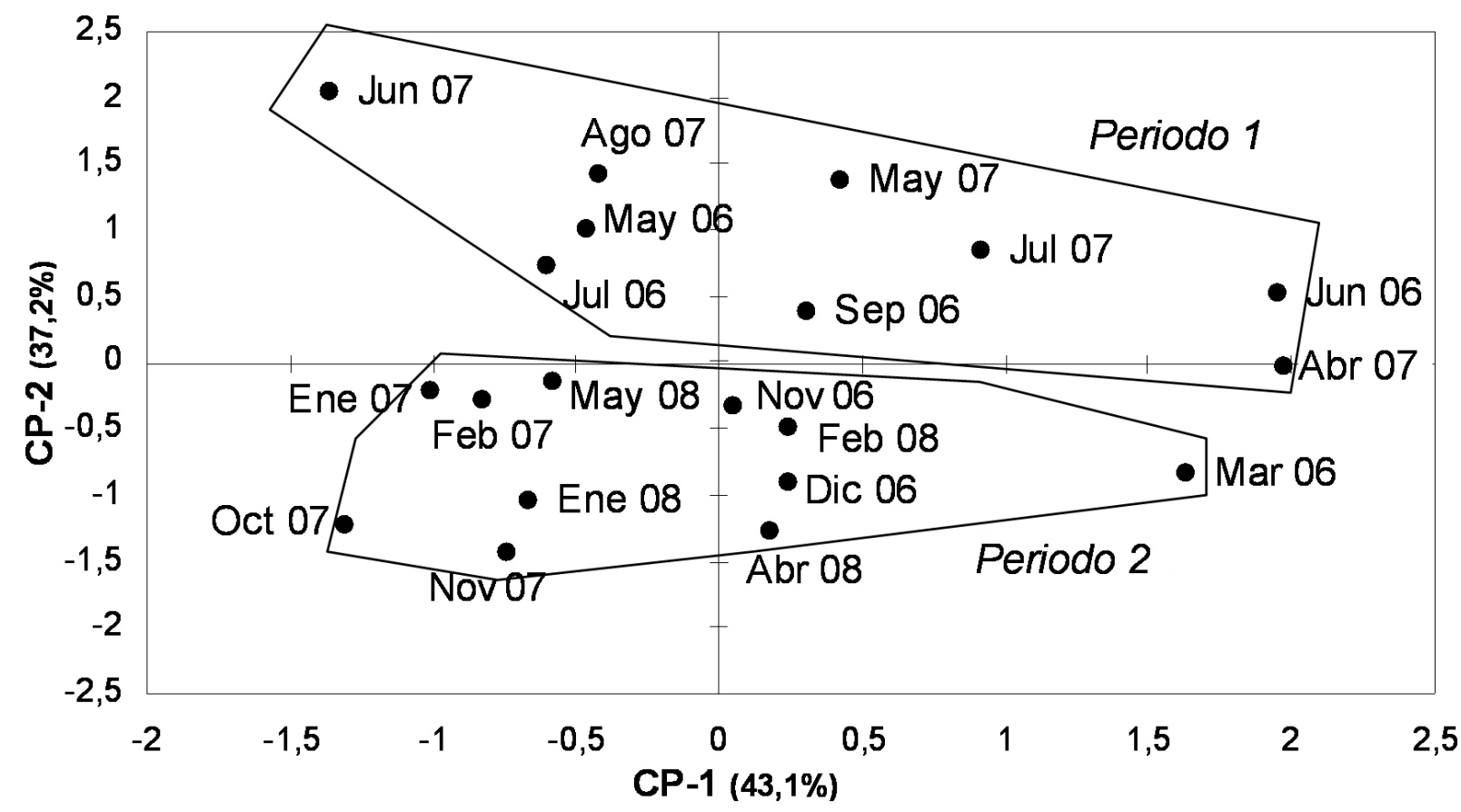

Figura 4. Ordenación y clasificación de las campañas de muestreo en los Periodos 1 (cálido-reclutamiento) y 2 (frío-no reclutamiento) en función de los resultados del Análisis de Componentes Principales.

Figure 4. Planning and classification of the sampling events in the periods 1 (warm-recruitment) and 2 (cold-non recruitment) according to the results of the Principal Components Analysis.

\begin{tabular}{llll}
\hline & $\begin{array}{c}\text { CPUE } \\
\text { machos }\end{array}$ & $\begin{array}{c}\text { CPUE } \\
\text { hembras }\end{array}$ & $\begin{array}{c}\text { CPUE } \\
\text { indeterminados }\end{array}$ \\
\hline Temperatura & $-0,241$ & $-0,240$ & $0,529^{*}$ \\
& $p=0,305$ & $p=0,308$ & $p=0,016$ \\
Conductividad & $-0,127$ & 0,023 & 0,365 \\
& $p=0,593$ & $p=0,922$ & $p=0,114$ \\
Salinidad & $-0,127$ & 0,028 & 0,364 \\
Recubrimiento & $p=0,595$ & $p=0,907$ & $p=0,115$ \\
& 0,200 & $-0,112$ & 0,217 \\
Profundidad & 0,132 & $-0,153$ & $-0,108$ \\
& $p=0,580$ & $p=0,518$ & $p=0,649$ \\
\hline
\end{tabular}

Tabla 1. Coeficientes de correlación de las variables del mesohábitat y las abundancias relativas (CPUEs) de Aphanius iberus en las Salinas de Marchamalo (*Coeficiente significativo, $\mathrm{p}<0,05$ ).

Table 1. Correlations coefficients between environmental variables and relative abundances (CPUEs) of Aphanius iberus in the Marchamalo wetland (*Significant coefficient, $\mathrm{p}<0,05$ ).

\begin{tabular}{lcc}
\hline & \multicolumn{2}{c}{ Componente } \\
& CP-1 (43,1\%) & CP-2 (37,2\%) \\
\hline Temperatura & 0,321 & 0,761 \\
Conductividad & 0,991 & 0,005 \\
Salinidad & 0,992 & 0,025 \\
Recubrimiento & 0,023 & 0,744 \\
Profundidad & $-0,283$ & 0,856 \\
\hline
\end{tabular}

Tabla 2. Coeficientes de correlación de las variables de mesohábitat con los componentes (CP-1 y CP-2) extraídos del Análisis de Componentes Principales (entre paréntesis: varianza explicada).

Table 2. Correlations coefficients between the mesohabitat variables and the components extracted from the Principal Components Analysis (in brackets: explained variance). 
2007). El grupo o fase temporal 2 incorpora principalmente campañas de otoño e invierno, momento en el que las capturas de indeterminados son escasas y corresponde con la época de no reclutamiento (noviembre de 2006 a febrero de 2007 y octubre de 2007 a mayo de 2008). En consecuencia, el estudio del efecto del microhábitat sobre la abundancia relativa de la especie se ha realizado de forma separada para datos obtenidos en la fase temporal 1 (Periodo cálido - Reclutamiento) y en la fase temporal 2 (Periodo frío - No reclutamiento) (Periodos 1 y 2 en Fig. 4).

El primer paso en el estudio del efecto del microhábitat fue el análisis de relaciones bivariantes entre la abundancia como variable dependiente (CPUE de machos, hembras e indeterminados) y las seis variables descriptoras del microhábitat. Con datos obtenidos de minnow-traps hemos detectado que las relaciones obtenidas entre la abun-
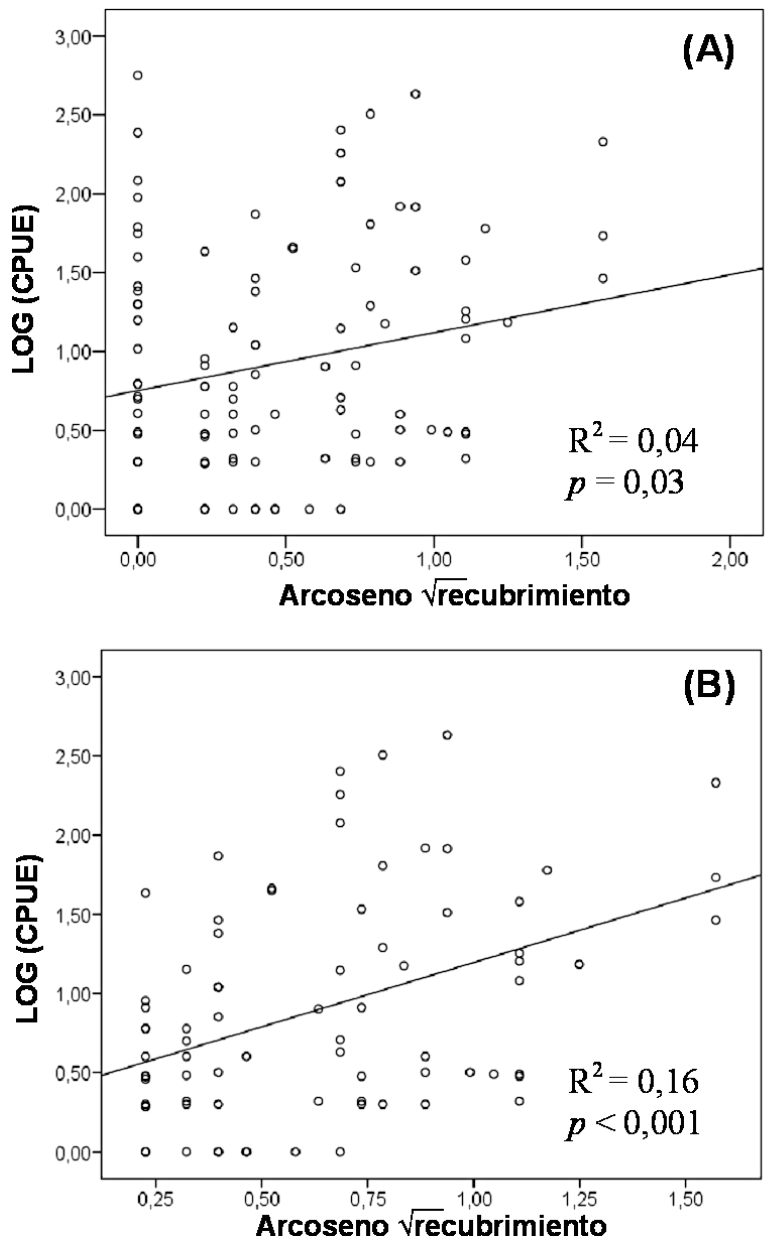

dancia y las variables relativas a la vegetación acuática (recubrimiento y volumen), se ven alteradas por las elevadas capturas obtenidas en unidades de muestreo sin vegetación (Fig. 5). Hemos observado que las trampas tipo minnow-trap ejercen cierta atracción por refugio en zonas sin vegetación sobre los individuos de la especie, por tanto, se optó por eliminar del análisis los datos procedentes de unidades de muestreo con recubrimientos inferiores al $5 \%$. No obstante, este efecto no se observó cuando el método de trampeo fue la trampa de polietileno, estableciendo en estos casos las regresiones con la totalidad de datos. Se encontraron relaciones lineales significativas entre la abundancia de adultos y las variables de vegetación (recubrimiento y volumen) en ambas fases temporales, y también con la profundidad en la fase temporal 1 (Periodo cálido - Reclutamiento).

Los indeterminados mostraron relaciones con
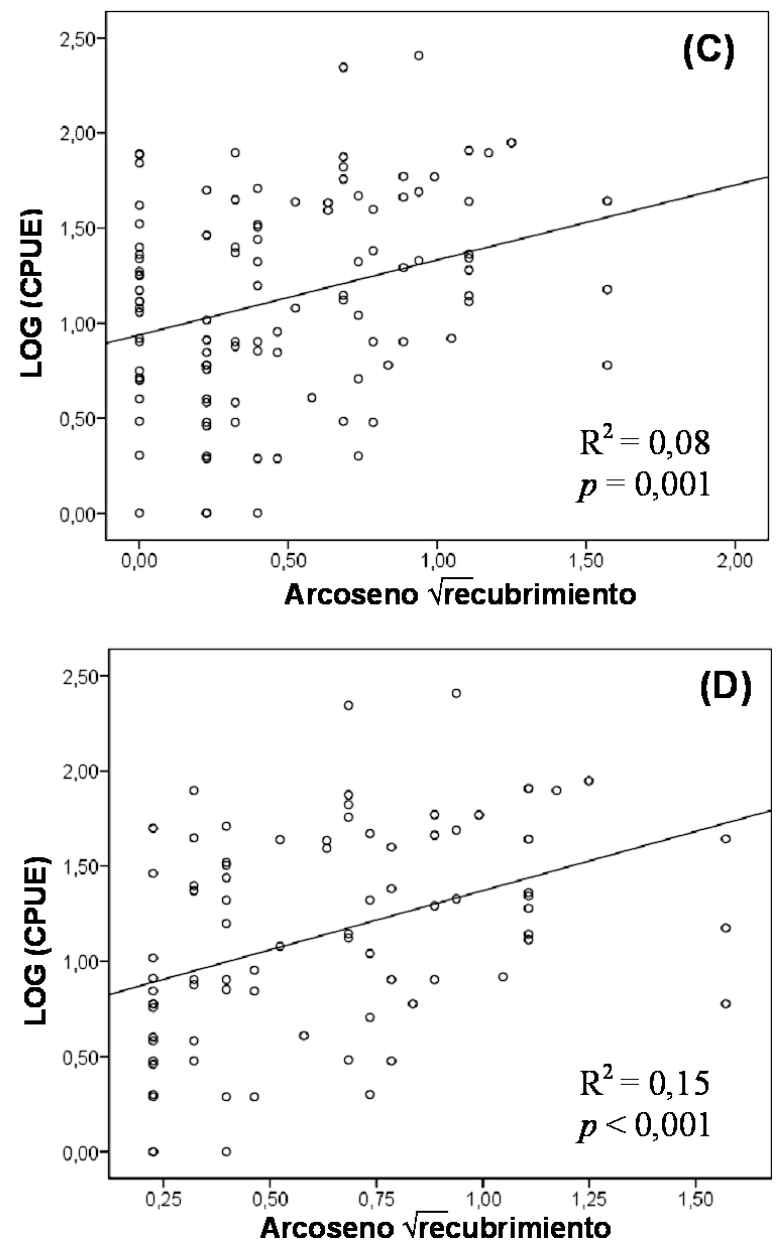

Figura 5. Relaciones entre recubrimiento de vegetación y abundancia de Aphanius iberus en el Periodo 1 (Cálido-Reclutamiento). A y C: datos con la totalidad de unidades de muestreo para machos y hembras. B y D: datos con la eliminación de unidades de muestreo con recubrimiento inferior al $5 \%$.

Figure 5. Relationship between vegetation cover and abundance of Aphanius iberus in the Period 1 (Warm-Recruitment). A and C: data from total sampling units for males and females. B and D: data from sampling without units with less than $5 \%$ vegetation cover. 
la vegetación durante la fase temporal 2, es decir, cuando son más escasos en la población se ven más afectados por la presencia de vegetación acuática.

No se han encontrado relaciones entre las abundancias de adultos capturados en las trampas de polietileno y las variables de microhábitat, a excepción del volumen de la vegetación que pre- senta un efecto significativo sobre ambos sexos e indeterminados en la fase temporal 2. En esta fase temporal, también se han encontrado relaciones entre la abundancia de indeterminados y el recubrimiento por vegetación.

En las tablas 3 y 4 se presentan los resultados obtenidos del análisis de regresión múltiple entre la abundancia de la especie (machos, hembras e

\begin{tabular}{|c|c|c|}
\hline $\begin{array}{c}\text { Modelo } \\
\text { Minnow-traps }\end{array}$ & Ecuación de regresión & \\
\hline $\begin{array}{l}\text { Machos } \\
\text { Volumen de vegetación } \\
\text { Profundidad } \\
\text { Distancia a la orilla }\end{array}$ & $\begin{array}{l}\log (\text { CPUE })=-1,947+0,039 \text { Volumen }^{2}+ \\
0,756 \text { Log }\left(\text { Profundidad }^{2}\right)+4,661(1 / \sqrt{ } \text { Distancia a la orilla })\end{array}$ & $\begin{array}{l}R^{2}=0,334 \\
F=11,728 ; g l=(1 ; 76) \\
p<0,001\end{array}$ \\
\hline $\begin{array}{l}\text { Hembras } \\
\text { Recubrimiento de vegetación } \\
\text { Volumen de vegetación } \\
\text { Profundidad }\end{array}$ & $\begin{array}{l}\text { Log }(\text { CPUE })=0,365 \text { Arcoseno }(\sqrt{ } \text { Recubrimiento vegetal }) \\
+0,018 \text { Volumen }^{2}+0,355 \text { Log }\left(\text { Profundidad }^{2}\right)\end{array}$ & $\begin{array}{l}R^{2}=0,225 \\
F=8,647 ; g l=(1 ; 76) \\
p=0,038\end{array}$ \\
\hline $\begin{array}{l}\text { Indeterminados } \\
\text { Volumen de vegetación }\end{array}$ & Log CPUE = 0,159 Volumen ${ }^{2}$ & $\begin{array}{l}R^{2}=0,043 \\
F=4,522 ; g l=(1 ; 78) \\
p=0,037\end{array}$ \\
\hline \multicolumn{3}{|l|}{ Trampas polietileno } \\
\hline $\begin{array}{l}\text { Hembras } \\
\text { Volumen de vegetación }\end{array}$ & Log CPUE $=0,188+0,017$ Volumen ${ }^{2}$ & $\begin{array}{l}R^{2}=0,078 \\
F=6,184 ; g l=(1 ; 60) \\
p=0,016\end{array}$ \\
\hline $\begin{array}{l}\text { Indeterminados } \\
\text { Recubrimiento de vegetación }\end{array}$ & Log CPUE = 0,711 Arcoseno ( $\sqrt{\text { Recubrimiento vegetal })}$ & $\begin{array}{l}R^{2}=0,269 \\
F=23,482 ; g l=(1 ; 60) \\
p<0,001\end{array}$ \\
\hline
\end{tabular}

Tabla 3. Modelos de regresión lineal múltiple significativos entre las variables de microhábitat y la abundancia de Aphanius iberus (CPUEs de machos, hembras e indeterminados como variables dependientes) obtenidos en el Periodo 1 (Periodo cálido - Reclutamiento). Datos presentados en función de la metodología de captura.

Table 3. Significant multiple regression models between the microhabitat variables and the abundance of Aphanius iberus (dependent variables) in the Period 1 (Warm - Recruitment). Data presented separately for each method of capture.

\section{Modelo}

\section{Minnow-traps}

\begin{tabular}{|c|c|c|}
\hline $\begin{array}{l}\text { Machos } \\
\text { Recubrimiento de vegetación }\end{array}$ & $\log (C P U E)=1,860$ Arcoseno $(\sqrt{ }$ Recubrimiento vegetal $)$ & $\begin{array}{l}R^{2}=0,398 \\
F=35,340 ; g l=(1 ; 51) \\
p<0,001\end{array}$ \\
\hline $\begin{array}{l}\text { Hembras } \\
\text { Recubrimiento de vegetación }\end{array}$ & $\log (\mathrm{CPUE})=1,703$ Arcoseno $(\sqrt{ }$ Recubrimiento vegetal $)$ & $\begin{array}{l}\mathrm{R}^{2}=0,323 \\
\mathrm{~F}=28,858 ; \mathrm{gl}=(1 ; 51) \\
\mathrm{p}<0,001\end{array}$ \\
\hline
\end{tabular}

Tabla 4. Modelos de regresión lineal múltiple significativos entre las variables de microhábitat y la abundancia de Aphanius iberus (CPUEs de machos, hembras e indeterminados como variables dependientes) obtenidos en el Periodo 2 (Frío - No Reclutamiento). Datos presentados en función de la metodología de captura.

Table 4. Significant multiple regression models between the microhabitat variables and the abundance of Aphanius iberus (dependent variables) in the Period 2 (Cold - Nonrecruitment). Data presented separately for each method of capture. 
indeterminados, variables dependientes) y el microhábitat. Estos resultados son mostrados en función de la metodología de captura (minnow-traps y trampa de polietileno) y según la fase temporal (Periodo Cálido - Reclutamiento; Periodo frío No reclutamiento).

En la fase temporal 1 (Periodo cálido - Reclutamiento), las variables que mostraron un efecto significativo sobre la abundancia relativa de machos obtenida mediante minnow-traps fueron el volumen de vegetación, profundidad de la lámina de agua y distancia a la orilla (Tabla 3). El índice de tolerancia obtenido para el modelo en que intervienen las tres variables es de 0,915 , mostrando la no colinealidad entre las mismas. En el caso de las hembras capturadas con esta metodología, las variables que se incluyen en el modelo son recubrimiento, volumen de vegetación y profundidad, con un índice de tolerancia de 0,943. Las CPUE de indeterminados obtenidas mediante minnowtraps están influenciadas por el volumen de la vegetación (Tabla 3). Los modelos obtenidos con capturas procedentes de trampas de polietileno muestran resultados significativamente diferentes, obteniendo modelos significativos únicamente para hembras e indeterminados (Tabla 3).

En la fase temporal 2 (Periodo frío - No Reclutamiento), únicamente se han obtenido modelos significativos para el caso de las abundancias de machos y hembras capturados con minnowtraps. Las densidades de ambos sexos resultaron dependientes del recubrimiento vegetal (Tabla 4).

\section{Discusión}

A. iberus presenta el patrón típico de ciclo de vida de especies de peces de vida corta caracterizado por una época de expansión reproductiva seguida de un periodo de muerte masiva tras el esfuerzo reproductor realizado (Fernández-Delgado et al. 1988, García-Berthou \& Moreno-Amich 1992).

En las Salinas de Marchamalo, la abundancia de $A$. iberus varía estacionalmente de manera significativa a lo largo del año, mostrando los valores máximos de abundancia de adultos al final del verano y principios de otoño en los dos ciclos estudiados. En el presente estudio, el pico de individuos adultos característico de las fases finales del periodo de reclutamiento (ver Oliva-Paterna et al. 2009) fue menor durante el segundo ciclo, coincidiendo con aumentos de los valores de salinidad y conductividad en el sistema. En términos genera- les, los primeros estadios de vida son mucho más sensibles a los factores físico-químicos de estrés que los individuos adultos de una población (Holliday 1969). Así por ejemplo, los rangos de tolerancia de estadios iniciales a factores como la salinidad del agua pueden ser determinantes para la reproducción exitosa de una especie, y se ha demostrado que los efectos deletéreos de la salinidad sobre los estadios tempranos resultan mucho más conspicuos durante las primeras semanas de vida (Bohlen 1999). En consecuencia, la ausencia de un incremento importante de individuos adultos en nuestra población durante el segundo ciclo, podría estar relacionada con una mayor mortalidad de individuos indeterminados justo en la fase previa a la diferenciación morfológica observándose, de este modo, una disminución en el reclutamiento hasta la etapa adulta.

En trabajos anteriores realizados en esta misma localidad se observó cómo los aumentos drásticos de salinidad, relacionados con decrementos en el nivel del agua de las balsas, afectaron negativamente a la especie. Estos incrementos de salinidad provocaron mortandades masivas afectando a distintos parámetros de su estrategia de vida en el sistema (Ruiz-Navarro et al. 2007, Oliva-Paterna et al. 2009). Efectos de cambios drásticos en la salinidad sobre la biología de ciprinodóntidos han sido puestos de manifiesto en diversos estudios (Nordlie et al. 1991, Plaut 2000, Yildirim \& Karacuha 2008, entre otros). En suma, el hecho de que durante el segundo ciclo de estudio la densidad de A. iberus no haya sido tan elevada como en el primero puede deberse a la elevada salinidad y conductividad alcanzada en el medio.

Los ambientes hipersalinos y las explotaciones salineras son hábitats muy importantes para la conservación de A. iberus (Oliva-Paterna et al. 2006). No obstante, el abandono de la actividad salinera de estos espacios puede provocar perjuicios para las poblaciones de la especie allí presentes. En el ámbito de las Salinas de Marchamalo, la gestión del sistema se ha visto alterada con el cese de la actividad salinera tradicional. Cabe destacar los problemas de entrada de agua a las salinas desde el Mar Menor a lo largo del periodo de estudio y en periodos anteriores, ya que el canal de entrada ha quedado obstruido periódicamente por la dinámica de erosión-sedimentación costera, lo que ha venido provocando los aumentos de salinidad antes aludidos (Ruiz-Navarro et al. 2007, OlivaPaterna \& Torralva 2008, Oliva-Paterna et al. 
2009). A. iberus tiene una estrategia de vida oportunista (sensu Vila-Gispert \& Moreno-Amich 2002) adaptada a los cambios drásticos de las variables ambientales, aspecto que le permite proliferar en un ambiente tan estresante. De hecho, los sistemas acuáticos con elevada salinidad están actuando como un refugio importante para la especie, ya que las poblaciones ahí establecidas reducen enormemente los efectos de competencia interespecífica con especies invasoras poco viables en dichos ambientes (ej. Gambusia holbrooki Girard, 1859) (Oliva-Paterna \& Torralva 2008).

De forma adicional, las condiciones térmicas de las Salinas de Marchamalo son moderadas durante todo el año, con una temperatura media superior a $20^{\circ} \mathrm{C}$ que, junto con la ausencia de periodos prolongados de temperaturas bajas, pueden ser factores causantes de la presencia de un reclutamiento prolongado con la detección de individuos indeterminados en la mayoría de muestreos del periodo de estudio.

Para la población en su conjunto, las abundancias relativas obtenidas durante los meses más fríos han sido notablemente más bajas que en los meses de altas temperaturas. No obstante, las capturas obtenidas durante los meses de bajas temperaturas probablemente no son reflejo de la densidad poblacional real, ya que el muestreo de tipo pasivo induce cierto sesgo en los resultados debido a la menor actividad que presentan los individuos (Lucas \& Baras 2000). Esto se puede traducir en valores subestimados de capturabilidad en épocas frías, aspecto que se ve muy influenciado por la etología de la especie de estudio.

A su vez, acorde con los resultados obtenidos previamente con especies muy próximas taxonómicamente (Clavero et al. 2006), en nuestro estudio se muestran diferencias en la capturabilidad en función de los diferentes métodos de trampeo. Los individuos de menor tamaño son capturados con mayor eficacia en las trampas de polietileno y los adultos con mayor talla mediante el uso de minnow-traps. Por este motivo, el uso de diferentes tipos de trampeo a la hora de estudiar el ciclo de vida y relaciones con el hábitat de esta especie, y otras similares, debe ser una metodología a evaluar. En nuestro estudio, las interpretaciones relativas a individuos indeterminados más concluyentes se han obtenido a partir de los datos obtenidos mediante trampas de polietileno (más eficaces en la captura de esta fracción poblacional). Por el contrario, en el caso de densidades de adultos, la mayor eficacia de las minnow-traps nos ha llevado a interpretar de forma más satisfactoria los resultados obtenidos mediante este método.

Las condiciones ambientales, el grado de actividad de los individuos y el momento del ciclo de vida en que se encuentre la población van a ser factores determinantes para explicar las relaciones de la especie con el hábitat (Matthews 1998). El estudio de las variables del hábitat en la balsa de muestreo (mesohábitat), junto con análisis del ciclo de vida en términos de abundancia relativa de la especie, nos ha permitido distinguir dos fases temporales con alta homogeneidad en las condiciones limnológicas y en la dinámica poblacional. Estas fases pueden afectar de forma significativa al grado de actividad de la especie y, en consecuencia, a la interpretación del efecto del microhábitat sobre su dinámica. La primera fase se ha identificado como Periodo cálido o de reclutamiento y se caracteriza por tener valores elevados de profundidad, temperatura y recubrimiento de vegetación, coincidiendo con los momentos de máximas densidades de indeterminados. Por otra parte, una segunda fase caracterizada por presentar menor profundidad de la lámina de agua, temperatura y recubrimiento vegetal, a su vez coincidente con bajas densidades de indeterminados, se ha identificado como Periodo frío o de no reclutamiento.

En el análisis del efecto del microhábitat sobre la abundancia de la especie, se observó cómo el posible efecto refugio o de atracción de individuos que pueden ejercer las trampas tipo minnow-trap, tiene consecuencias sobre las relaciones existentes entre la abundancia y variables de microhábitat descriptoras del refugio para peces (ej. recubrimiento de vegetación). El efecto de atracción puede relacionarse con el diseño de las minnow-traps que, al estar recubiertas por una malla fina de color verde oscuro, pueden ofrecer sombra y simular un acúmulo subacuático de vegetación. Sin embargo, en el caso de las trampas de polietileno, al ser transparentes y de menor tamaño, es probable que no provoquen el mismo efecto de atracción. Este posible efecto de refugio de las minnowtraps fue corregido antes de incluir los datos en los modelos de regresión múltiple.

El estudio realizado mediante relaciones múltiples entre las abundancias de la especie y las variables del microhábitat ha mostrado resultados 
significativos en ambos periodos, tanto en el cálido o de reclutamiento como en el frío de no reclutamiento. Los resultados muestran mayor precisión, en términos de explicación de la varianza, durante la época fría que durante la época cálida. Esto debe estar influenciado por el grado de actividad de los peces, durante el verano la actividad de los individuos es mayor y esto se puede traducir en desplazamientos constantes en búsqueda de alimento, establecimiento de territorios o búsqueda de zonas de puesta (Alcaraz et al. 2008). En cambio, durante el invierno los individuos tienden a presentar menor grado de actividad con la consiguiente reducción de desplazamientos y, por tanto, las relaciones con las variables de microhábitat se muestran más robustas.

Estudios previos sobre el microhábitat de Aphanius baeticus Doadrio, Carmona \& Fernandez-Delgado, 2002 reflejan cómo esta especie muestra una selección de hábitat dependiente de la profundidad y la presencia de refugio (Clavero et al. 2005). En el caso de A. iberus se ha observado que las zonas con vegetación presentan mayor densidad de peces (Alcaraz et al. 2008, VerdiellCubedo et al. 2012). Según los resultados obtenidos en este estudio, las variables de microhábitat que han determinado la variación de abundancia relativa de adultos en el periodo cálido han estado relacionadas con el volumen y recubrimiento de vegetación acuática, la profundidad y la distancia a la orilla, es decir, variables que en su totalidad pueden relacionarse con la disponibilidad de refugio y zonas propensas para la puesta. Durante este periodo, la población presente en las Salinas de Marchamalo se encuentra en su fase de reproducción (Oliva-Paterna et al. 2009) y la especie hace uso de la vegetación para realizar las puestas (Martínez \& Cortés 1993), aspectos que probablemente explican el efecto significativo de la vegetación acuática sobre su abundancia. Además, en el caso de especies de peces de pequeña talla presentes en humedales someros, la vegetación provee de un refugio básico frente a predadores externos (ej. Charadriformes) (Rozas \& Odum 1988). Aunque la variación de profundidad en el sistema es escasa, la relación de la abundancia de la especie con esta variable, y también con la proximidad a las orillas conformadas por motas con presencia de Arthrocnemum spp. o Sarcocornia spp., también podría tener cierta relación con el aumento del refugio. Por otra parte, los resultados de las regresiones múltiples en la época fría tam- bién vienen determinados por relaciones significativas con la vegetación en todos los casos (adultos e indeterminados). Es probable que la microdistribución de la especie durante este periodo esté condicionada por la vegetación debido al refugio que otorga y también por la cantidad de alimento disponible en épocas frías (Rozas \& Odum 1988, Alcaraz \& García-Berthou 2007).

A modo de recomendación general, cabe destacar que una gestión de las Salinas de Marchamalo sin tener en cuenta criterios biológicos puede provocar acontecimientos ambientales con efectos perjudiciales sobre el ciclo de vida de $A$. iberus. En este sentido, una gestión hidrológica del sistema que conduzca a la eliminación de incrementos drásticos y rápidos en la salinidad de las balsas puede beneficiar la dinámica poblacional de la especie. Los aspectos negativos de estas crisis de salinidad han sido expuestos en trabajos previos realizados en este mismo humedal (Oliva-Paterna 2006, Ruiz-Navarro et al. 2007, Oliva-Paterna \& Torralva 2008, Oliva-Paterna et al. 2009). Además, los resultados obtenidos en el estudio del uso de hábitat nos permiten destacar que tanto la proliferación de praderas de vegetación sumergida como la presencia de motas salineras naturalizadas con vegetación o construidas con materiales que ofrezcan refugio (pequeñas escolleras sueltas, empalizadas, etc.), pueden ser vitales para el mantenimiento de poblaciones de la especie objetivo.

\section{Agradecimientos}

Mostrar nuestro agradecimiento a Asunción Andreu y Antonio García por su participación en las tareas de campo relacionadas con el estudio. Al Departamento de Zoología y Antropología Física de la Universidad de Murcia por proveer del entorno adecuado para la elaboración del trabajo. Los revisores del trabajo han mejorado notablemente las primeras versiones del mismo.

El trabajo de campo fue realizado en el marco del Proyecto LIFE-Naturaleza Conservación de stocks genéticos de Aphanius iberus en la Región de Murcia. Parte del estudio se ha realizado en el contexto del Máster en Gestión de la Biodiversidad de Ambientes Mediterráneos de la Universidad de Murcia.

\section{Referencias}

Alcaraz C \& García-Berthou E. 2007. Food of an endan- 
gered cyprinodont (Aphanius iberus): ontogenetic diet shift and prey electivity. Environmental Biology of Fishes 78: 193-207.

Alcaraz C, Pou-Rovira Q \& García-Berthou E. 2008. Use of a flooded salt marsh habitat by an endangered cyprinodontid fish (Aphanius iberus). Hydrobiologia 600: 177-185.

Araguas RM, Roldán MI, García-Marín JL \& Pla C. 2007. Management of a gene diversity in the endemic killifish Aphanius iberus: Revising Operational Conservation Units. Ecology of Freshwater Fish 16(2): 257-266.

Bohlen J. 1999. Influence of salinity on early development in the spined loach. Journal of fish biology 55 : 189-198.

Caiola N \& De Sostoa A. 2005. Possible reasons for the decline of two native toothcarps in the Iberian Peninsula: evidence of competition with the introduced Eastern mosquitofish. Journal of Applied Ichthyology 21: 358-363.

Casas JJ, Sánchez-Oliver JS, Sanz A, Furné M, Trenzado $C$, Juan $M$, Paracuellos $M$, Suárez $M D$ Fuentes F, Gallego I, Gil C \& Ramos-Miras JJ. 2011. The paradox of the conservation of an endangered fish species in a Mediterranean region under agricultural intensification. Biological Conservation 144(1): 253-262.

Clavero M, Blanco-Garrido F, Zamora L \& Prenda J. 2005. Size-related and diel variations in microhabitat use of three endangered small fishes in a Mediterranean coastal stream. Journal of Fish Biology 67: 72-85.

Clavero M, Blanco-Garrido F \& Prenda J. 2006. Monitoring small fish population in streams: A comparison of four passive methods. Fisheries Research 78(2-3): 243-251.

Clavero M, Blanco-Garrido F \& Prenda J. 2007. Population and microhabitat effects of interespecific interactions on the endangered Andalusian toothcarp. Environmental Biology of Fishes 78: 173-182.

Doadrio I. 2002. Atlas y Libro Rojo de los Peces Continentales de España. Dirección General de Conservación de la Naturaleza. Secretaría General de Medio Ambiente. Ministerio de Medio Ambiente.

Elvira B. 1998. Impact of introduced fish on the native freshwater fish fauna of Spain. En: Stocking and Introduction of Fish. Cowx IG (Ed). Fishing News Books, Oxford.

Esteve MA, Caballero JM, Jiménez A, Aledo E, Baraza F, Guirao J, Robledano F \& Torres A. 1995. Los paisajes del agua en la región de Murcia. Caracterización ambiental y perspectivas de gestión de los humedales. En: Agua y futuro en la Región de Murcia. Asamblea Regional de Murcia.

Fernández-Delgado $C$, Hernando J, Herrera $M$ \& Bellido M. 1988. Age, growth and reproduction of Aphanius iberus (Cuv. \& Val., 1846) in the lower reaches of the Guadalquivir river (south-west Spain). Freshwater Biology 20: 227-234.

Fernández-Delgado C, Torralva M, Oliva-Paterna FJ \& Pintos R. 1999. Caracterización ecológica del hábitat del fartet (Lebias ibera, Valenciennes 1846) en una pequeña cuenca hidrográfica del bajo Guadalquivir. En: Planelles M (Ed.). Peces Ciprinodóntidos Ibéricos: Fartet y Samaruc. Monografía. Generalitat Valenciana. Valencia.

Fouilland E \& Fossati O. 1996. Trapping efficiency of plastic bottle wickertraps for population assessment of river Macrobrachium (Crustacea: Decapoda). Fisheries Research 28: 343-351.

García-Berthou E \& Moreno-Amich R. 1992. Age and growth of an Iberian cyprinodont, Aphanius iberus (CUV. \& VAL.), in its most northerly population. Journal of Fish Biology 40: 929-937.

Harrison TD, Ramm AEL \& Cerff EC. 1986. A low-cost effective trap for use in sampling aquatic fauna. Aquaculture 58: 145-149.

Holliday FGT. 1969. The effects of salinity on the eggs and larvae of teleost. En: Fish Physiology. Volume 1. Hoar WS \& DJ Randall (Eds.). Academic Press. Orlando.

Lucas M \& Baras E. 2000. Methods for studying spatial behaviour of freshwater fishes in the natural environment. Fish and Fisheries 1: 283-316.

Martínez E \& Cortés E. 1993. A fondo: Aphanius iberus. Acqua 3: 5-10.

Matthews WJ. 1998. Patterns in Freshwater Fish Ecology. Chapman \& Hall. New York.

Nordlie FG, Walsh SJ, Haney DC \& Nordlie TF. 1991. The influence of ambient salinity on routine metabolism in the teleost Cyprinodon variegatus. Journal of Fish Biology 38: 115-122.

Oliva-Paterna FJ. 2006. Biología y conservación de Aphanius iberus (Valenciennes, 1846) en la Región de Murcia. Tesis Doctoral. Departamento de Zoología y Antropología Física. Universidad de Murcia.

Oliva-Paterna FJ, Torralva M \& Fernández-Delgado C. 2006. Threatened fishes of the World: Aphanius iberus (Cuvier \& Valenciennes 1846) (Cyprinodontidae). Environmental Biology of Fishes 75: 307-309.

Oliva-Paterna FJ \& Torralva M. 2008. El fartet en la Región de Murcia: biología y conservación. Consejería de Ordenación del Territorio y Medio Ambiente. Dirección General del Medio Natural. Murcia.

Oliva-Paterna FJ, Ruiz-Navarro A, Torralva M \& Fernández-Delgado C. 2009. Biology of the endangered cyprinodontid Aphanius iberus in a saline wetland (SE Iberian Peninsula). Italian Journal of Zoology 76(3): 316-329.

Quinn GP \& Keough MJ. 2002. Experimental designs a data analysis for biologist. Cambridge University Press. UK.

Plaut I. 2000. Resting metabolic rate, critical swimming speed, and routine activity of the eurihaline cyprinodontid, Aphanius dispar, acclimated to a wide range of salinities. Physiological and Biochemical Zoology 73(5): 590-596.

Rincón PA, Correas AM, Morcillo F, Risueño P \& LobónCerviá J. 2002. Interaction between the introduced eastern mosquitofish and two autochthonous Spanish toothcarps. Journal of Fish Biology 61(6): 15601585.

Rozas LP \& Odum WE. Occupation of submerged aquatic vegetation by fishes: testing the roles of food 
and refuge. Oecologia 77: 101-106.

Ruiz-Navarro, Oliva-Paterna FJ \& Torralva M. 2007. Somatic condition of Aphanius iberus (Valenciennes 1846) in Marchamalo wetland (Mar Menor; SE Spain): Effects of management. Anales de Biología 29: 53-59.

UICN 2012. IUCN Red List of Threatened Species. Version 2012.2. <www.iucnredlist.org>. Downloaded on 31 October 2012.

Verdiell-Cubedo D, Torralva M, Ruiz-Navarro A \& OlivaPaterna FJ. 2012 Fish assemblages in different littoral habitat types of a hypersaline coastal lagoon
(Mar Menor, Mediterranean Sea). Italian Journal of Zoology. (Versión online).

Vila-Gispert A. \& Moreno-Amich R. 2002. Life-history patterns of 25 species from European freshwater fish communities. Environmental Biology of Fishes 65: 387-400.

Visauta-Vinacua B. 1997. Análisis estadístico con SPSS para Windows. McGraw-Hill. Madrid.

Yildirim Ö \& Karakuha A. 2008. Effect of salinity on growth performance and survival rate of Aphanius chantrei (Galliard, 1895). Journal of Applied Ichthyology 24: 345-347. 